University of Montana

ScholarWorks at University of Montana

10-2003

\title{
Evolution of Avian Locomotion: Correlates of Flight Style, Locomotor Modules, Nesting Biology, Body Size, Development, and the Origin of Flapping Flight
}

Kenneth P. Dial

University of Montana - Missoula, kdial@mso.umt.edu

Follow this and additional works at: https://scholarworks.umt.edu/biosci_pubs

Part of the Biology Commons

Let us know how access to this document benefits you.

\section{Recommended Citation}

Dial, Kenneth P., "Evolution of Avian Locomotion: Correlates of Flight Style, Locomotor Modules, Nesting Biology, Body Size, Development, and the Origin of Flapping Flight" (2003). Biological Sciences Faculty Publications. 214.

https://scholarworks.umt.edu/biosci_pubs/214

This Article is brought to you for free and open access by the Biological Sciences at ScholarWorks at University of Montana. It has been accepted for inclusion in Biological Sciences Faculty Publications by an authorized administrator of ScholarWorks at University of Montana. For more information, please contact scholarworks@mso.umt.edu. 


\title{
The Auk \\ A Quarterly \\ Journal of Ornithology \\ Vol. 120 No. 4 October 2003
}

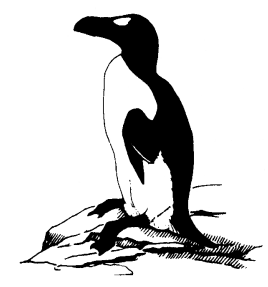

The Auk 120(4):941-952, 2003

PERSPECTIVES IN ORNITHOLOGY

\section{EVOLUTION OF AVIAN LOCOMOTION: CORRELATES OF FLIGHT STYLE, LOCOMOTOR MODULES, NESTING BIOLOGY, BODY SIZE, DEVELOPMENT, AND THE ORIGIN OF FLAPPING FLIGHT}

\author{
KenNeth P. DiaL ${ }^{1}$ \\ Flight Laboratory, Avian Studies Program, Division of Biological Sciences, University of Montana, \\ Missoula, Montana 59812, USA
}

Avian nesting STRATEGies, flight style, hatchling and adult body size, developmental precocial-altricial trajectories, and the origin of flight are frequently studied as separate topics rather than addressed as integrated and mutually dependent phenomena. Consider the following questions: What is the evolutionary basis of the precocial-to-altricial developmental spectrum observed among birds? Does flight proficiency correlate with aspects of nesting biology among extant avian species? Are there reasonable alternative hypotheses to the popular arboreal-cursorial dichotomy regarding the origin of avian flight? In an attempt to address the above questions, I offer a model based on locomotion and life history that integrates nesting biology, flight capacity, body mass, morphological modularity, and stage at hatching among extant avian clades. Acknowledging that those variables are neither independent nor mutually exclusive, I suggest that the range and tendency for each factor be compared simultaneously and within an integrated matrix. Using a broad-brush approach, I provide a first approximation of a synthetic viewpoint for evaluating locomotor and life-history features of birds. In addition, phylogenetic correlates are

\footnotetext{
${ }^{1}$ E-mail: kdial@selway.umt.edu
}

discussed in hope of stimulating future studies that test that thesis among living and extinct clades. I begin with a brief review of each of the five criteria embedded in the model.

\section{The Five Variables}

\section{LOCOMOTOR MODULES}

Morphological determinants of mobility among avian taxa can be viewed as a composite of three discrete locomotor modules (forelimbs, hindlimbs, and tail), which are differentially elaborated according to specializations in lifestyle and have independent neuromuscular control patterns that are used in different modes of locomotion (takeoff, steady flapping flight, walk-run) (Gatesy and Dial 1993, 1996).

In basal, nonvolant cursorial avian taxa (e.g. ratites), mass of the hindlimbs dominates and acts as the primary locomotor machinery, whereas forelimbs are highly reduced and functionally vestigial during locomotion. Cursorial ground birds that do fly (e.g. Galliformes, Tinamiformes) possess massive hindlimbs composed of mostly aerobic muscle (fastoxidative glycolytic fibers), whereas their flightfunctional forelimbs are powered by anaerobic muscle (fast-glycolytic fatiguable fibers) and are recruited during brief, explosive escape bouts. 


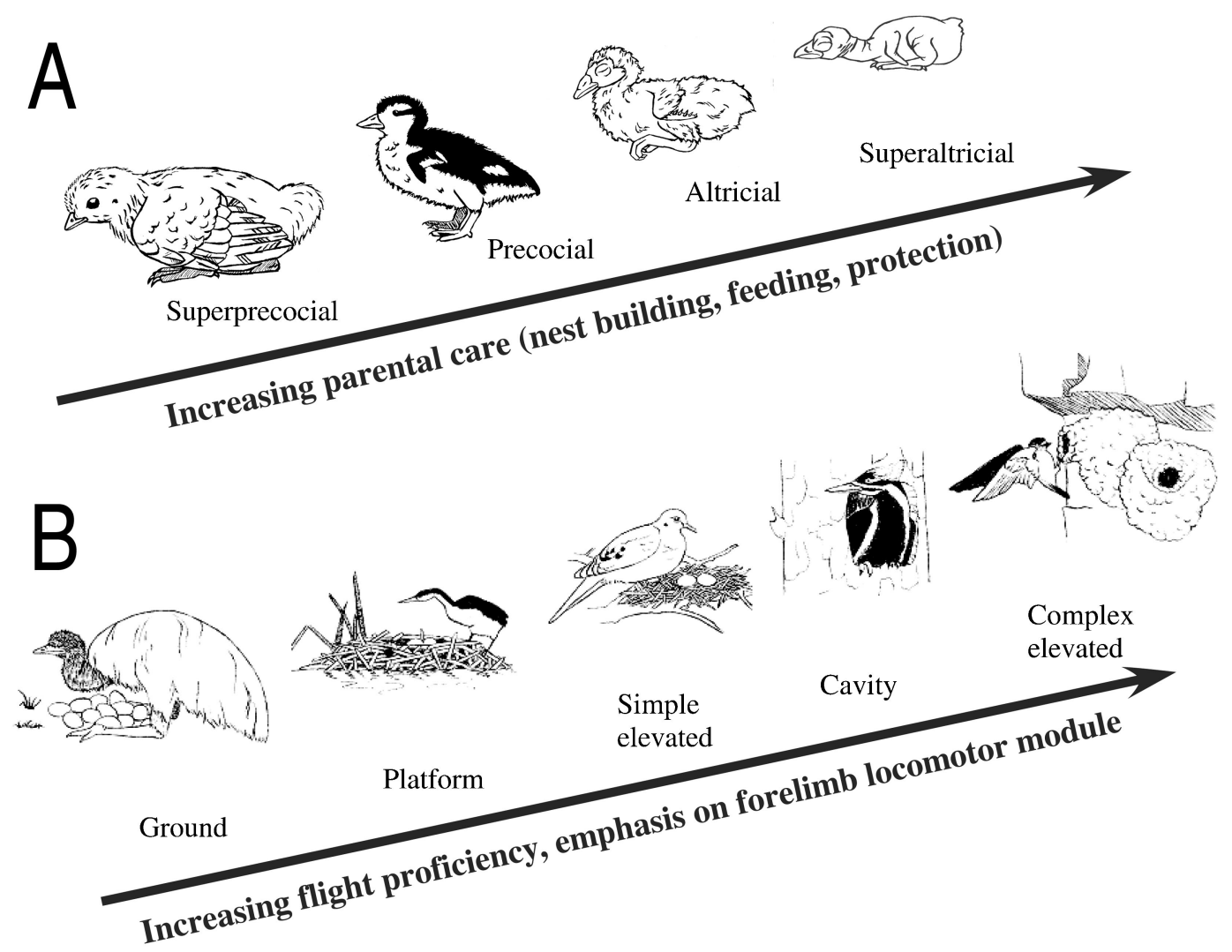

FIG. 1. (A) Spectrum of developmental stages from super-precocial brush turkeys to super-altricial songbirds. Note: There is a concomitant increase in parental care with increasing altricial development. (B) Nest building diversity from simple-ground to complex-elevated. Note: There is an increase in flight capability among taxa raised within a complex and elevated nest, requiring significant parental care.

In noncursorial taxonomic groups that possess substantial hindlimb mass (e.g. Anseriformes, Gruiformes, Gaviiformes, Ciconiiformes), flight styles are noticeably restricted by poor maneuverability and a narrow range of flight speeds. Passerines have features consistent with maximal flight performance that include small body size (i.e. high power-to-mass ratios), significant forelimb investment (i.e. large wing and shoulder muscles), sophisticated neuromuscular control of broad surface areas (wings and tail) (Dial 1992), and dietary exploitation of high-energy fuel sources (Calder 1984, Schmidt-Nielsen 1984, Norberg 1990). At the final extreme, birds that are strongly forelimb dominated (e.g. Apodiformes, Hirundinidae) have diminutive hindlimbs and are highly restricted in their terrestrial locomotor abilities; some are unable even to walk. That mosaic modular locomotor arrangement, observed in birds in general and Passeriformes in particular, is suggested to be responsible for promoting avian diversification and specialization (Fig. 1A) (Gatesy and Dial 1996).

\section{Developmental Spectrum and Parental Care}

The precocial-altricial developmental spectrum ranges from megapodes whose hatchlings resemble adult birds and can fly on the day that they hatch, to passeriformes, whose hatchlings are nearly embryonic (i.e. hatching naked, blind, thermally dependent, and essentially immobile) (Ricklefs 1976, 1983; Starck and Ricklefs 1998) (Fig. 1A). The spectrum recognizes a broad array of developmental stages: superprecocial, 
three categories of precocial species, semiprecocial, two semialtricial, altricial, and superaltricial (Nice 1962, Starch 1993).

Parental care necessarily varies with the different categories of development at hatching (Nice 1962, Skutch 1976, Starck and Ricklefs 1998, Martin et al. 2000, Remeš and Martin 2002). Precocial and superprecocial birds are characterized by patterns of simple parental care, minimal nest attendance, and simple nest structure; all those features are considered phylogenetically primitive. Galliformes and Anseriformes seek their own food the day that they hatch but depend on parents for some degree of brooding and protection. On the other hand, altricial species are characterized by sophisticated parental care that includes complex nest building and high attendance to the offspring. Those traits are associated with altricial development (e.g. complex nest construction and strong parental care) and are also correlated with an increase in range of flight styles, flight speeds, and ecological habits.

\section{Nesting Biology}

Nest building among living birds varies from a simple accumulation of materials on the ground to elaborately constructed refuges positioned upon secluded and elevated substrates (Skutch 1976, Collias and Collias 1984, Oniki 1985, Hansen 2000) (Fig. 1B). Although not widely recognized, nest construction and placement are correlated with other features such as flight ability. For example, basal avian taxa (ratites and many Galliformes) create a simple depression in the ground to harbor their incubating eggs, similar to those preserved from nonavian dinosaurs (Fig. 1B) (Horner and Makela 1979, Horner 1982, Norell et al. 1995, Varricchio et al. 1997). The progression of nest construction complexity moves from cryptically placed ground nests of some galliforms to simple elevated nests, as observed in Columbiformes, Cuculiformes, and Ciconiiformes. Taxa that construct an elevated nest within a bush or tree or upon a cliff or rock ledge tend to be better fliers than most simple ground nesters. Young raised in elevated and cavity nests, including primary (Psittaciformes, Piciformes, and Coraciformes) and secondary (many Passeriformes) cavity nesters, have a robust forelimb flight apparatus, and tend to de-emphasize their hindlimb mass, which is again consistent with increasing flight capacity (Gatesy and Dial 1996, Warrick 1998). As nest placement (e.g. invisibility, inaccessibility), construction (e.g. impregnability, camouflage), and attendance (e.g. feeding, protection, incubation) increase in complexity, we observe a concomitant enhancement of flight styles, including maneuverability and acceleration, requiring a wider range of locomotor ability. The most complex and derived nest construction is associated with some Passeriformes, particularly swallows, oropendolas, and weaver finches. Weaver finches (Ploceidae, Passeridae) and oropendolas (Icteridae) build intricately woven chambered, pendant nests hung from the resilient thin branches of bushes and trees in predator-rich environments. Perhaps the most predator-proof nests are those of swallows (Hirundinidae) and swifts (Apodidae) that frequently construct mud encasements secured to the most remote overhanging feature within their habitat (e.g. cliffs and numerous humanmade structures).

\section{Body SIZE}

Birds span over five orders of magnitude in body size; extant species range from the $5 \mathrm{~g}$ Cuban Bee Hummingbird (Mellisuga helenae) to the Ostrich (Struthio camelus), which exceeds $150 \mathrm{~kg}$. Although numerous studies have recognized the importance of body size to physiology (Calder 1984, Schmidt-Nielsen 1984, Brown and West 2000), ecology (McMahon and Bonner 1983, Peters 1983, Brown and West 2000), and aspects of life history (Marzluff and Dial 1990, Roff 1992), minimal attention has been directed toward empirical testing of allometric correlates of locomotor performance (acceleration, deceleration, maneuverability, and range of flight speeds). Maneuvering and linear acceleration are functions of mass-specific power (Warrick 1998) transferred to lift and thrust; however, our ability to measure the relationship between body mass and mechanical power output (Pennycuick 1975) remains a challenge (but see Dial et al. 1997, Tobalske et al. 2003). In addition, the significance of adverse scaling of lift and power output must also be resolved if we are to clearly understand the mechanisms underlying the basic relationship of body size and locomotor performance (Ellington 1991, Marden 1994, Askew et al. 2001). Future studies 
that carefully quantify locomotor performance (e.g. power-to-mass and power-to-lift ratios), both among and within members of a clade, will prove invaluable in elucidating new trends in foraging behavior, habitat use, predator-prey relationships, and additional ecological patterns among animal communities.

How an animal exploits its threedimensional environment is largely based on the relationship among its mass, properties of its locomotor machinery (e.g. muscle investment and fiber types), and density of the medium in which they travel (Fig. 2). Despite differences in flight style, the largest masses attained by all extant flying birds converge on $\sim 12 \mathrm{~kg}$ (e.g Mute Swan [Cygnus olor], Kori Bustard [Ardeotis kori], Wandering Albatross [Diomedea exulans],
Wild Turkey [Meleagris gallopavo], and Andean Condor [Vultur gryphus]). That upper mass figure for flying birds should not be considered coincidental (Pennycuick 1969, 1985). Mechanical power required to fly is determined by muscle force output and contraction frequency and is predicted to scale nearly independent of body mass $\left(\mathrm{M}^{0}-\mathrm{M}^{1 / 6}\right)$, but available power scales negatively to the one-third body mass $\left(\mathrm{M}^{-1 / 3}\right)$ (Ellington 1991, Marden 1994) (Fig. 2). The point at which the power slopes intersect appears to match the mass region of the upper limit (12-14 kg) (Fig. 2). The limited power available for flight relates to the power required in large birds and likely limits their flight performance. At the other end of the spectrum, we see that smaller animals, as a rule, enjoy an excess of

\section{Power-to-Mass Ratio}

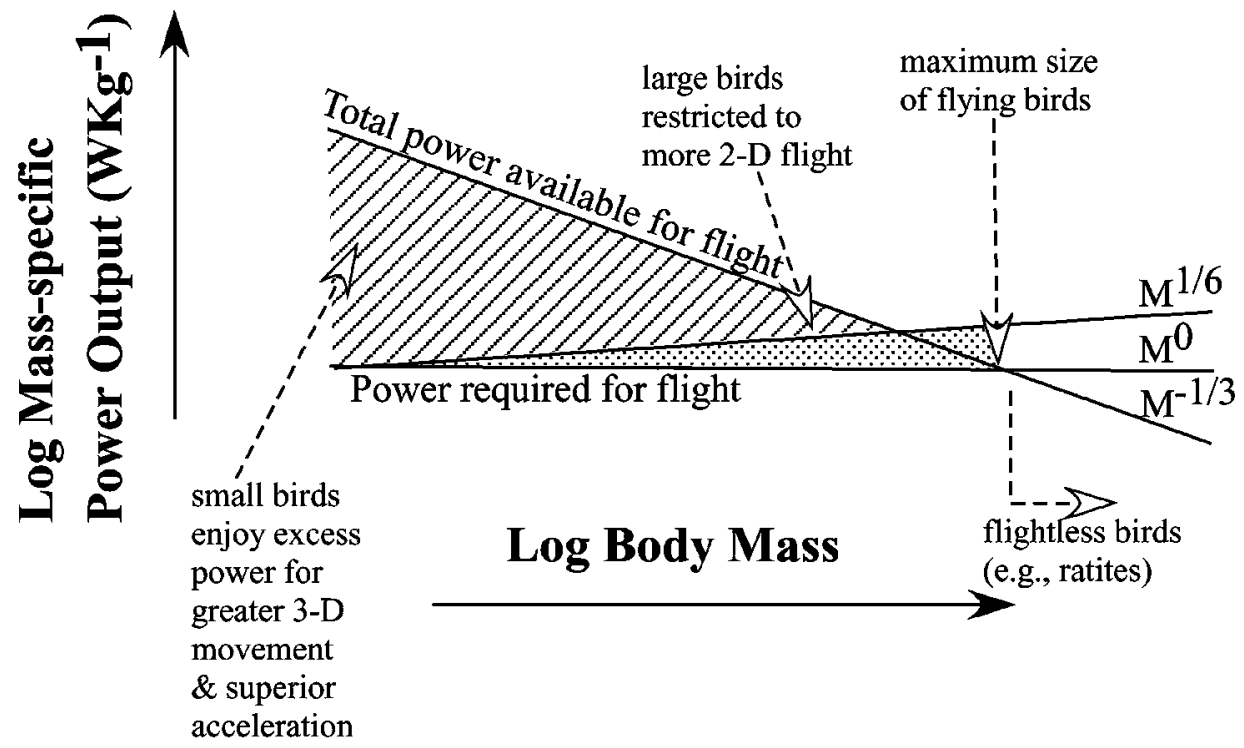

FIG. 2. Theoretical relationship between body size and power output among birds. Power required is thought to scale independent $\left(\mathrm{M}^{0}\right)$ or slightly positive $\left(\mathrm{M}^{1 / 6}\right)$ among bird of differing sizes. Power available is thought to scale negatively $\left(\mathrm{M}^{-1 / 3}\right)$. One explanation for that trend is that wing-beat frequency (WBF) scales to the negative one-third power as do most oscillating entities, such as a series of swinging pendulums of different lengths. Empirical data of the available or required power in birds requires further investigation. Nevertheless, a trend does exist where small birds possess an excess of muscle power output relative to their body mass and, therefore, are able to enjoy a more three-dimensional world in time and space. Because power is a product of work per unit time, the slower WBF of larger species restricts them to proportionally less power output. The largest extant flying species are approximately 12-14 kg body mass (Pennycuick 1989); that is thought to represent the intersection of the power required and power available curves. All species exceeding $12-14 \mathrm{~kg}$ are flightless (e.g. ratites). 
available power output, translating into greater flight capabilities. Therefore, most small species inhabit more three-dimensional space and enjoy greater accelerative performance than their larger relatives and predators. I argue that the power-to-mass ratio (i.e. high wing-beat frequency and muscle force generation) in small birds is an unappreciated but important determinant of taxonomic diversity. Species with superior locomotor performance in time and space are able to unlock numerous ecological and evolutionary opportunities.

\section{FLight StYles}

The range and diversity of flight abilities among birds is extraordinary (Savile 1957, Rayner 1988, Norberg 1990, Warrick 1998). Body mass alone does not determine flight performance. Specific morphological attributes permit increased maneuverability and linear acceleration. Generally, species that are intrinsically maneuverable have large wings relative to body mass, and species that are extremely maneuverable are capable of generating high mass-specific power at slow speeds by creating large force asymmetries between the two wings (Warrick 1998). At one end of the locomotor spectrum are the nonvolant birds that, in addition to the ratites, include species of cormorants, parrots, pigeons, waterfowl, and rails. All have reduced forelimb muscle masses and enlarged (relative to their closest flying relatives) hindlimb modules. Short-burst fliers (e.g. Galliformes and Tinamiformes) may have robust flight muscles, but flight abilities are limited by their muscle physiology (fatiguable, anaerobic, fast-glycolytic muscle fibers that exhaust quickly with respect to power output; Schorger 1966, Crabtree and Newsholme 1972, Marden 1994, Tobalske and Dial 2000). Some taxa exhibit limited flight styles and are restricted to a narrow range of flight speeds: some fly rapidly (e.g. Anseriformes, Gaviiformes, Podicipediformes, many Charadriiformes) and others relatively slowly (e.g. Ciconiiformes, Phoenicopteriformes). The most speciose clade of birds, the Passeriformes, exhibit very good to excellent maneuvering skills and a broad range of flight speeds and styles (including aerial coursing insectivores such as swallows, and hawking aerial insectivores such as tyrannid flycatchers; Warrick 1998)_as well as a diversity of hindlimb locomotor capabilities used in concert with flight (e.g. ground and tree gleaners). Contrary to the passeriformes, which may owe much of their maneuverability to small size and thus inherently high massspecific power and low wing loading (Warrick et al. 1998), larger species of other taxa also display and may be ecologically defined by high maneuverability (e.g. frigatebirds [Frigatidate] and acciptiters [Accipitridae]). In terms of maneuverability, the highest performance fliers are probably the coursing aerial insectivores (e.g. swifts, swallows, nighthawks, and diminutive falcons; Warrick 1998). All in that group possess small body sizes and high aspect-ratio wings; some (e.g. swifts) also have relatively small wings (high-speed wings; Savile 1957). Members within each flight-style group possess a distinct suite of traits with respect to nesting biology, relative body size, developmental stage at hatching, and particular emphasis on specific locomotor modules.

\section{Five-PARAmeter Examination}

By simultaneously inspecting tendencies of various natural- and life-history phenotypic traits among extant avian taxa, I predict that new and integrative patterns will emerge regarding ecological strategies and evolutionary trajectories of that diverse clade (Fig. 3). When taxa are assigned a position within the graph by simultaneously considering the five character states, an interesting trend emerges (Fig. 3). Basal extant species possess the following characters: relatively large body size, superprecocial to precocial young, locomotor morphology dominated by the hindlimb module, minimal parental care, flightlessness or only brief bursts of flight, and simple ground nests (e.g. ratites, megapodes, most Galliformes, and some Anseriformes). Members within the next taxonomic cluster have relatively large body size, precocial young, narrow flight styles, ground or platform nests, and increased propensity for parental care (e.g. Podicipediformes, Gruiformes, Gaviiformes, and some Anseriformes). Representatives of the next cluster have medium-to-large body size, a relatively balanced state of locomotor modules (tail, hindlimb, and forelimb), simply constructed nests elevated in vegetation or upon geological structures, altricial young, increased parental care (feed- 


\section{Five-way Comparison}

\section{Nesting Types}

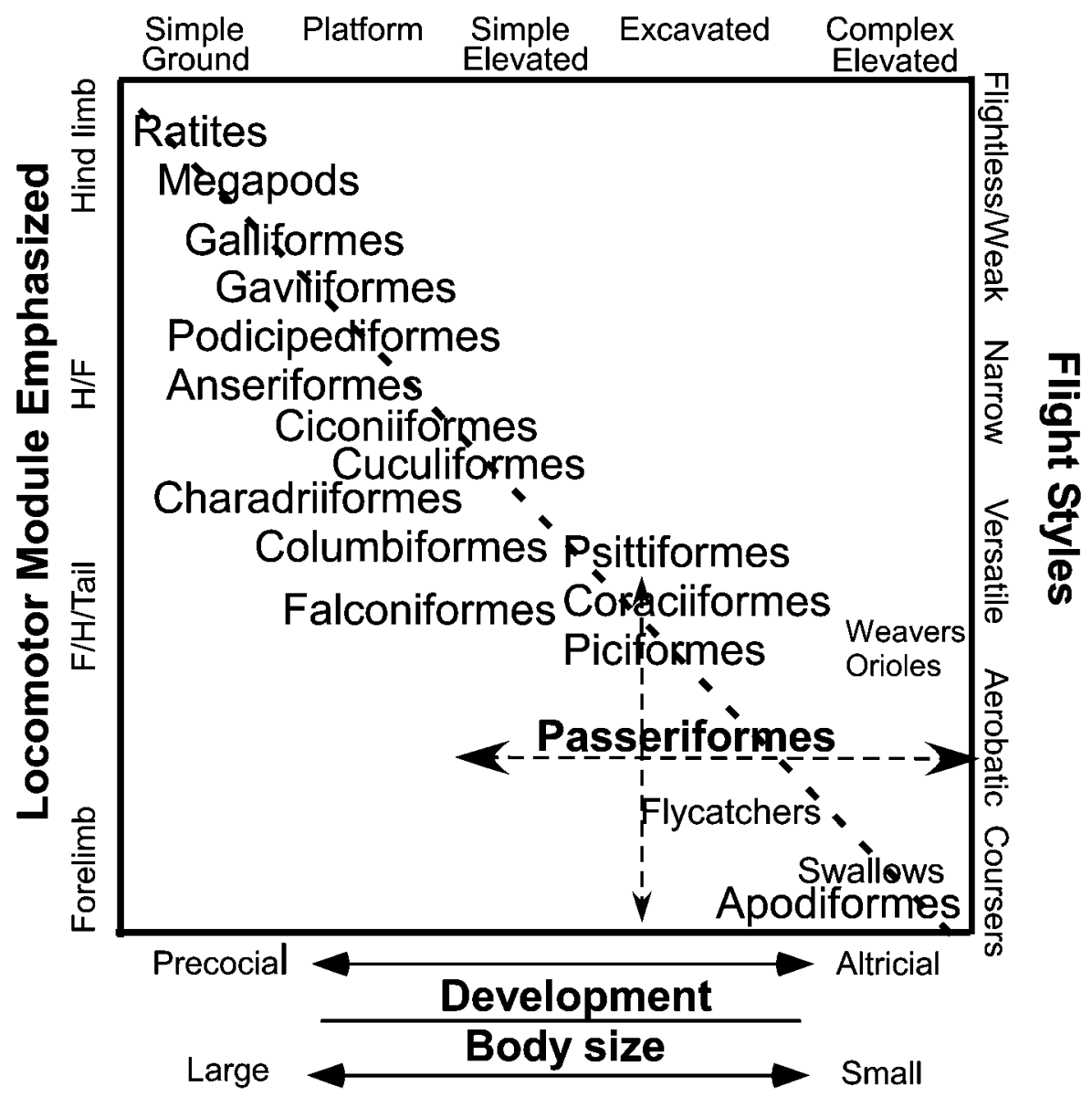

FIG. 3. Five-way comparison of variables associated with avian locomotion and evolution of life-history traits. Note the general trend in the suite of traits associated with primitive bipedal cursors toward the aerial cursors. Members within the passerine group exhibit the greatest diversity in form and flight styles (adapted from Dial 2003b).

ing, protection, incubation), and pronounced investment in the forelimb flight machinery and hence with wider flight styles (e.g. Columbiformes, Ciconiiformes, Falconiformes). At the end of that locomotor spectrum are the specialized "aerial coursers" (e.g. Apodidae, Hirundinidae, Caprimulgiformes, Fregatidae, and Phaethontidae) endowed with extraordinary flight capabilities (Warrick 1998). Those taxa are usually characterized by relatively small body size (thus high power-to-mass ratios); atrophic or highly specialized (e.g. strong grasping digits) hindlimb modules; pronounced forelimb musculoskeletal investment; elaborately constructed nests, secluded nests, or both; intense parental care; and superaltricial young. With respect to those groups on the continuum, the Passeriformes are the most speciesrich. In general, individuals within that clade possess small body size, pronounced forelimb investment (but also retain substantial hindlimb and tail modules), broad feeding and nesting habits, intense parental care, and altricial to superaltricial young. 
When living birds are broadly surveyed and assigned a categorical position within that five-way scheme, a noticeable trend emerges among basal to derived groups (Fig. 3). The more derived species (both across and within broad taxa) tend to sequester young in a confined nest and offer relatively greater parental care compared to more primitive relatives. That in turn permits a developmental trajectory toward reduced hindlimb and increased forelimb investment for increased flight capacity (Fig. 3). I argue that a positive feedback relationship existed during the history of avian flight such that an increase in complexity of nest construction and parental care correlated with an augmentation in forelimb investment and a concomitant decrease in hindlimb investment. That trajectory enhanced use of habitat space as small, forelimb-dominated species continued to exploit more ecological space (e.g. evasive insects, small fruits and seeds on the terminus of thin branches of bushes and trees); at the same time, those same species continue to enjoy greater predator-escape performance through superior horizontal and vertical acceleration.

The suite of morphological traits associated with basal avian taxa appears consistent with the limited paleontological material of protobird theropods. Those animals were bipedal (hindlimb dominated), relatively large (compared to their avian relatives), reared precocial young, and offered rudimentary to moderate forms of parental care (e.g. Horner and Makela 1979, Horner 1982, Norell et al. 1995, Varricchio et al. 1997). Such features are particularly consistent for Caudipteryx, which also possessed rudimentary wings (Norell et al. 2002, Ji et al. 1998). In addition, that taxon exhibits all of the expected features of an animal capable of employing wing-assisted incline running (Dial 2003). We require significantly more information on various outgroups to Neornithes (e.g. among the Enantiornithes) if we are to shed light on that subject.

\section{Antipredation Escape Vector and The Origin of Avian Flight}

To interrelate trends among the variables discussed, I pose that predation pressure, historical and present, be considered a primary selective agent that drove the observed patterns. The importance of predation as a major selective force that structured a variety of phenotypic traits cannot be overemphasized (Martin 1988, 1995; Conway and Martin 2000; Martin et al. 2000; Remeš and Martin 2002). Although more work is required to fully elucidate historic trends in avian and theropod developmental patterns (Horner et al. 2001, Padian et al. 2001), it has been suggested that precocial development was primitive and altricial development derived (Cracraft 1981, 1988; Sibley and Ahlquist 1990; Starck and Ricklefs 1998).

As we continue to study the locomotor development of precocial avian species (e.g. Galliformes and Tinamiformes), new insight into the behaviors elicited by the more basal extant and extinct taxa will unfold (Dial 2003). For example, a previously unappreciated yet common behavior of many ground birds (e.g. Galliformes) is their tendency to get off the ground and seek an elevated refuge (e.g. trees, rocks, cliffs, etc.) when not foraging for food. To that end, juveniles and adults of four gallinaceous species studied to date employ their flapping protowings or fully developed wings to create aerodynamic forces directed toward the substrate rather than skyward, so that their hindlimbs power the animal up to the refuge. The wings of those species are used like spoilers on a racecar to increase foot traction (Bundle and Dial 2003, Dial 2003). Wing-assisted incline running resolves many of the inconsistencies inherent within the historic cursorial-arboreal debate (e.g. Ostrom 1974, 1979; Hecht et al. 1985; Padian 1986; Feduccia 1996) by offering an alternative and testable hypothesis for the adaptive significance of intermediate wing forms (Bundle and Dial 2003, Dial 2003).

I propose that the incipiently feathered forelimbs of small, bipedal protobirds provided the same locomotor advantages for inclined running as in extant birds. Because ground birds exhibit the common tendency to elevate themselves off the ground to reduce predation risk in ecological time, that behavioral propensity may have been relevant to protobirds in evolutionary time. And if antipredation tactics, as directed by locomotor strategies, significantly structure bird biology, we should expect regular co-varying trends among the variables discussed.

Progress is underway to move beyond the intractable and irresolvable cursorial-arboreal debate by studying evolution of the wing stroke (Padian and Chiappe 1998; Padian 2001; Dial and 
Bundle 2003; Dial 2003). Future studies of the origin and evolution of avian flight will greatly benefit from those willing to integrate diverse disciplines and complement the traditional paleontological and aerodynamic theoretical efforts. Our rich knowledge of extant birds, their behavior, life-history, ecology, and ontogeny have only minimally been used to explore alternative hypotheses on the origin of flight.

\section{Forelimb-dominated Aerial Species and} Predation Pressure

All forelimb-dominated taxa, regardless of their phylogenetic position (from tropicbirds to swifts, penguins to nighthawks), share a suite of life-history and behavioral traits, the most obvious goal is to raise their young in predation-safe environments. Forelimb-dominated species tend to possess nonmobile, altricial, and therefore highly vulnerable young, and are thus forced to locate nesting sites that are essentially free of predators. Some forelimb-dominated species resort to ground nesting but execute extraordinary migrations by flying over vast oceans to raise their young on predator-free and remote islands (e.g. Procellariiformes, many Charadriiformes). Thus, forelimb-dominated taxa have figuratively jettisoned their excess hindlimb baggage and thus are particularly vulnerable to predation during development. Not surprisingly, those same species are predisposed to local decimation or extinction when exposed to introduced predators, particularly humans or other exotic species.

\section{Future TEsting}

By arranging most ordinal clades onto a diagram of evolutionary trends (Fig. 4), based on grouping the character states of the variables considered in the "five-way test" (Fig. 3), we can visualize a systematic association from the more basal to derived avian groups. The diagram is certainly incomplete and is not intended to be interpreted as a phylogeny. However, independent phylogenetic analyses of the component taxa (e.g. Felsenstein 1985, Harvey and Pagel 1991, Cracraft and Clarke 2001) will clearly show that many of those trends phylogenetically change more or less in lockstep, and that when groups have departed from their normal phylogenetic habits in one respect, they generally adopt other correlated features.
Extrapolating that trend to consider possible suites of life-history traits among avian ancestors may provide insight into the evolution of basal avian features. This article, however, is not intended to address all of the current issues in paleontological discussions (e.g. theropod growth rates, degree of parental care in extinct species, degree of flight capabilities in primitive avian taxa), nor is it intended to argue that altricial development and more active flight styles were absent in other theropod taxa (e.g. enantiornithines and confuciusornithines that exhibit elaborate flight machinery). It is important, however, that future studies consider the obvious trends and central tendencies among suites of life-history, ecological, and behavioral traits because that may provide insight into unappreciated strategies available among extinct clades.

Taxa that depart from the general pattern created by the five-way comparison should exhibit corresponding differences in the other variables. There are many examples of secondarily acquired transitions for all variables discussed. For example, some species of Passeriformes nest on the ground, thus resorting to a more primitive nesting style but with a new suite of survivorship features. My model suggests that such groups should have tendencies toward the following: small body masses (i.e. yielding high power-to-mass ratios for explosive and maneuverable escape flight), some degree of early hindlimb function in the young, sequestering of young in a highly cryptic nest (essentially under vegetation or substrate), complex brooding and feeding behaviors (secretive), and relatively faster developmental rates. From another perspective, members of a clade that exhibit large differences in adult body size (e.g. Anseriformes and Galliformes ranging from 0.2 to $12 \mathrm{~kg}$ ) should exhibit directional tendencies among the variables discussed, which suggests a coordinated linkage among those traits. Teal are capable of vertical takeoff and flight whereas swans struggle to become airborne over a $50 \mathrm{~m}$ horizontal distance and then attain only shallow climb rates. The alcids, considering their wing-propelled swimming and island-refuge nesting, represent an excellent group to explore life-history character trait evolution. They have much longer duration of offspring development relative to other Charadriiformes. Is the correlation of wing-propelled diving and their derived 

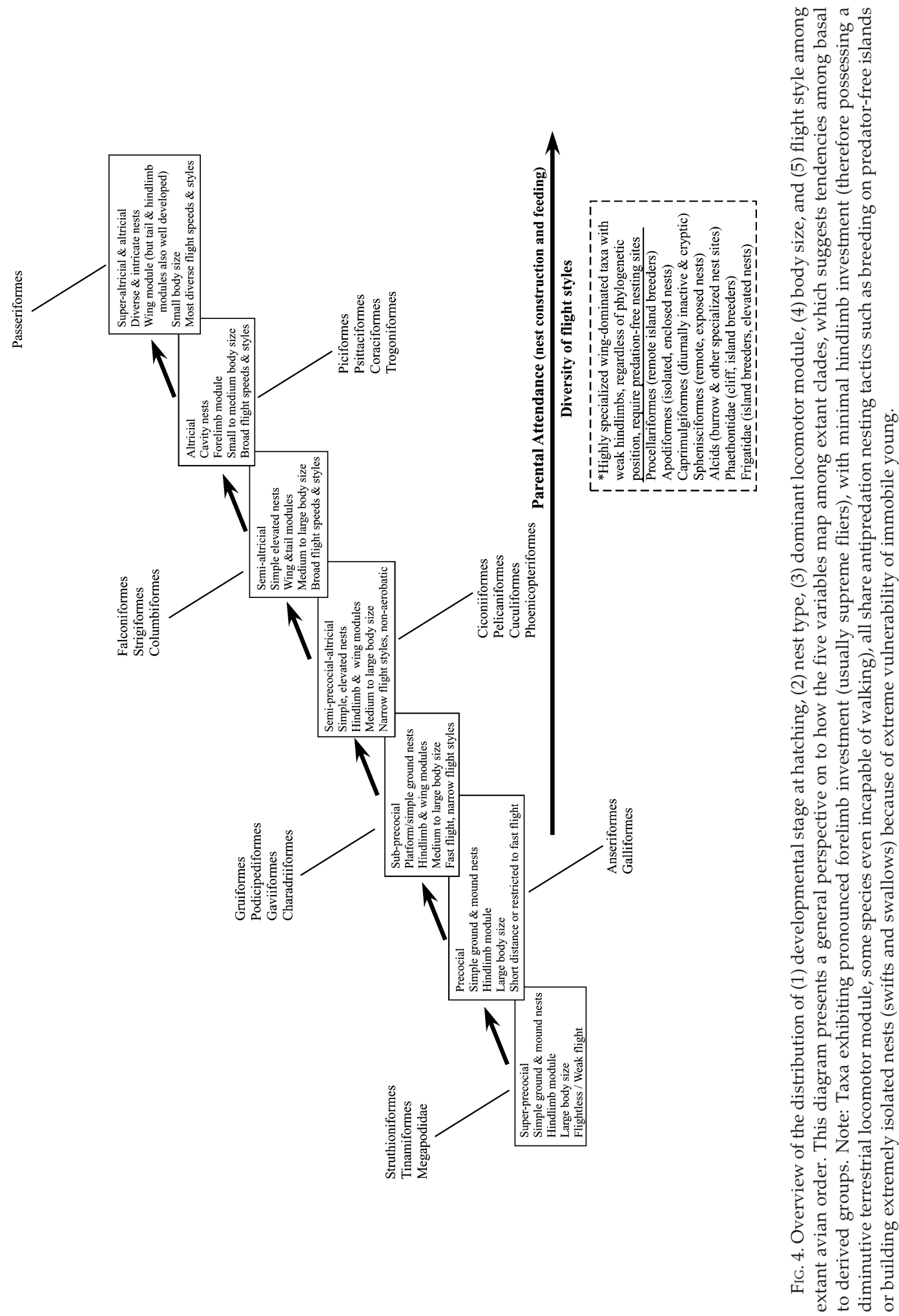
hole-cliff nesting accidental or causal? Some avian groups are expected to deviate from the general model presented here, but I suggest that their departure will provide novel insight into the distinctiveness of their habitat, phylogenetic history, or both.

Various authors have recently offered separate evaluations of each of the five variables discussed (e.g. evolution of parental care, Carey and Adams [2001]; life history and mating systems, Bennett and Owens [2002]; phylogenetic survey of developmental stages, Cracraft [1988] and Starck and Ricklefs [1998]; body size relations, Brown and West [2000]), but here the attempt has been to complement and integrate those efforts by simultaneously comparing multiple life-history variables within an integrated matrix and explain why the five features considered here collectively co-vary.

We should be able to test the model presented by two independent means. First, one can provide an ecological test, for example, by randomizing the character states of the variables and determining if any birds exist that fill the vast majority of randomized syndromes. A prediction would follow that there will be few that fit that condition, yet any exceptions will reveal interesting strategies. A phylogenetic test would also be appropriate. If there are deviations from the presented syndrome, one should expect departures to be secondarily derived with inevitable phylogenetic signals emerging from the investigation. That condition can be inspected by reviewing features of the taxon from which the deviant bird group evolved. The hypothesis is that the unusual features of the deviant will be found in its ancestral group. Finally, inspecting trends in avian locomotor modularity may enhance our understanding of the complex trends within avian life-history biology (e.g. developmental, reproductive), behavioral ecology (e.g. foraging strategies, habitat selection, community structure), and paleontology.

I suggest that selection on physiological and morphological features that affect locomotor performance has influenced patterns of speciation, species diversity, community structure, and animal behavior in more predictive ways than previously appreciated. Future exploration into the ecology, origin, and evolution of avian biology may benefit from a more integrated and synthetic approach by considering correlated trajectories of body size, nesting behavior, flight performance, and locomotor modules.

SUMMARY

Among vertebrates, birds possess a unique modular anatomic arrangement that permits different modes of locomotion powered by regionally specific musculoskeletal apparati (forelimbs, hindlimbs, and tail). Morphological investment emphasizing either the hindlimb or forelimb module varies predictably with trends in (1) developmental hatchling stage (i.e. precocial-altricial spectrum), (2) body size, (3) flight style (e.g. weak, narrow- and broad-speed range, and aerobatic fliers), and (4) parental care (e.g. nest construction and placement, feeding and protection of young). Lineages that evolve advanced flight skills deemphasize their hindlimb module and invest heavily in their forelimb module. Hatchling immobility correlates with a de-emphasis of hindlimb tissue and augmentation of the forelimb-flight apparatus, thus leading to an increase in flight proficiency. An increase in parental care correlates with that shift from hindlimb to forelimb locomotion because the nest-bound young require provisioning and refuge (e.g. a complexly constructed, elevated nest). Body size, developmental stage at hatching, flight style, nesting biology, and locomotor modularity appear to be intimately related and change in concert within and among clades.

\section{ACKNOWLEDGMENTS}

A special thanks is extended to T. E. Martin and K. Padian for their input during various stages of this project. I thank M. W. Bundle, H. Davis, B. E. Jackson, J. M. Marzluff, R. L. Hutto, S. M. Gatesy, E. Greene, R. Prum, B. W. Tobalske, and D. R. Warrick for discussions and comments on the manuscript. B. Peluso illustrated the birds and nests in Figure 1. This research was supported by the National Science Foundation.

\section{Literature Cited}

Askew, G. N., R. L. Marsh, and C. P. Ellington. 2001. The mechanical power output of the flight muscles of Blue-breasted Quail (Coturnix chinensis) during take-off. Journal of Experimental Biology 204:3601-3619.

Bennett, P. M., And I. P. F. OWens. 2002. Evolutionary Ecology of Birds: Life Histories, 
Mating Systems, and Extinction. Oxford University Press, New York.

Brown, J. H., AND G. B. West. 2000. Scaling in Biology. Oxford University Press, New York

Bundle, M. W., And K. P. Dial. 2003. Mechanics of wing-assisted incline running. Journal of Experimental Biology. In press.

Carey, J. R., AND J. Adams. 2001. The preadaptive role of parental care in the evolution of avian flight. Archaeopteryx (Eichstätt) 19:97-108

Calder, W. A. 1984. Size, Function, and Life History. Harvard University Press, Cambridge, Massachusetts.

Chiappe, L. M., And L. M. Witmer. 2002. Mesozoic Birds: Above the Heads of Dinosaurs. California University Press, Berkeley.

Collias, N. E., AND E. C. Collias. 1984. Nest Building and Bird Behavior. Princeton University Press, Princeton, New Jersey.

Conway, C. J., And T. E. Martin. 2000. Evolution of avian incubation behavior: Influence of food, climate and nest predation. Evolution 54:670-685.

Crabtree, B., and E. A. Newsholme. 1972. The activities of phosphorylase, hexokinase, phosphofructokinase, lactate dehydrogenase and the glycerol 3-phosphate dehydrogenases in muscles from vertebrates and invertebrates. Biochemical Journal 126:49-58.

Cracraft, J. 1981. Toward a phylogenetic classification of the recent birds of the world (Class Aves). Auk 98:681-714

Cracraft, J. 1988. The major clades of birds. Pages. 339-361 in The Phylogeny and Classification of the Tetrapodes, vol. 1 (M. J. Benton, Ed.). The Systematics Association Special Volume 35A, Oxford University Press, New York.

Cracraft, J., and J. Clarke. 2001. The basal clades of modern birds. Pages 143-156 in New Perspectives on the Origin and Early Evolution of Birds: Proceedings of the International Symposium in Honor of John H. Ostrom (J. Gauthier and L. F. Gall, Eds.). Peabody Museum of Natural History, Yale University, New Haven, Connecticut.

DiAL, K. P. 1992. Activity patterns of the wing muscles of the pigeon (Columba livia) during different modes of flight. Journal of Experimental Zoology 262:357-373.

DiAL, K. P. 2003a. Wing-assisted incline running and the evolution of flight. Science 299: 402-404.

DiAL, K. P. 2003b. Locomotor modularity: Patterns among flight style, nesting biology, development, and body size. In Proceedings of the 23rd International Ornithological Congress, Beijing, China. In press.

Dial, K. P., A. A. Biewener, B. W. Tobalske, And D.
R. WARRICK. 1997. Mechanical power output of bird flight. Nature 390:67-70.

ElLington, C. P. 1991. Limitations on animal flight performance. Journal of Experimental Biology 160:71-91.

Feduccia, A. 1996. The Origin and Evolution of Birds. Yale University Press, New Haven, Connecticut.

Felsenstein, J. 1985. Phylogenies and the comparative method. American Naturalist 125:1-15.

Gatesy, S. M., And K. P. Dial. 1993. Tail muscle activity patterns in walking and flying pigeons (Columba livia). Journal of Experimental Biology 176:55-76.

Gatesy, S. M., and K. P. Dial. 1996. Locomotor modules and the evolution of avian flight. Evolution 50:331-340.

Hansell, M. H. 2000. Bird Nests and Construction Behaviour. Cambridge University Press, United Kingdom.

Harvey, P. H., and M. D. Pagel. 1991. The Comparative Method in Evolutionary Biology. Oxford University Press, Oxford.

Hecht, M. K., J. H. Ostrom, G. Viohl, and P. Wellnhofer, EDS. 1985. The Beginnings of Birds. Freunde des Jura-Museums, Eichstätt, Germany.

HorNer, J. R. 1982. Evidence of colonial nesting and "site fidelity" among ornithatchiandinosaurs. Nature 297:675-676.

Horner, J. R., and R. MaKela. 1979. Nest of juveniles provides evidence of family structure among dinosaurs. Nature 282:296-298.

Horner, J. R., A. J. de Ricelès, And K. Padian. 2001. Comparative osteohistology of some embryonic and perinatal archosaurs: Phylogenetic and behavioral implications for dinosaurs. Paleobiology 27:39-58.

Ji, Q., P. J. Currie, M. A. Norell, AND S.-A. Ji. 1998. Two feathered theropods from the Upper Jurassic/Lower Cretaceous strata of northeastern China. Nature 393:753-761.

Marden, J. H. 1994. From damselflies to pterosaurs: How burst and sustainable flight performance scale with size. American Journal of Physiology 266:R1077-R1084.

Martin, T. E. 1988. Processes organizing opennesting bird assemblages: Competition or nest predation. Evolutionary Ecology 2:37-50.

MARTIN, T. E. 1995. Avian life history evolution in relation to nest sites, nest predation, and food. Ecological Monographs 65:101-127.

Martin, T. E., P. R. Martin, C. R. Olson, B. J. Heidinger, and J. J. Fontaine. 2000. Parental care and clutch sizes in North and South American birds. Science 287:1482-1485.

Marzluff, J. M., and K. P. Dial. 1990. Life history correlates of taxonomic diversity. Ecology 72: 428-439. 
McMahon, A. T., AND J. T. Bonner. 1983. On Size and Life. Scientific American Books, New York.

NicE, M. M. 1962. Development of behavior in precocial birds. Transactions of the Linnaean Society of New York 8:1-211.

Norberg, U. M. 1990. Vertebrate Flight. SpringerVerlag, New York.

Norell, M. A., J. M. Clark, L. M. Chiappe, and D. DashzeveG. 1995. A nesting dinosaur. Nature 378:774-776.

Norell, M. A., Q. Ji, K. GaO, C. Yuan , Y. Zhao, AND L. WANG. 2002. Palaeontology: Modern feathers on a non-avian dinosaur. Nature 406: 36-37.

ONIKI, Y. 1985. Why robin eggs are blue and birds build nests: Statistical tests for Amazonian birds. Ornithological Monographs 36:536-545.

Ostrom, J. H. 1974. Archaeopteryx and the origin of flight. Quarterly Review of Biology 49:27-47.

Ostrom, J. H. 1979. Bird flight: How did it begin? American Scientist 67:46-56.

PAdian, K., ED. 1986. The Origin of Birds and the Evolution of Flight. Memoirs of the California Academy of Sciences, no. 8.

PADIAN, K. 2001. The false issues of bird origins: An historiographic perspective. Pages 485499 in New Perspectives on the Origin and Early Evolution of Birds: Proceedings of the International Symposium in Honor of John H. Ostrom (J. Gauthier and L. F. Gall, Eds.). Peabody Museum of Natural History, Yale University, New Haven, Connecticut.

Padian, K., And L. M. Chiappe. 1998. The origin of birds and their flight. Scientific American 278: $38-47$.

Padian, K., A. J. de Ricelès, and J. R. Horner. 2001. Dinosaurian growth rates and bird origins. Nature 412:405-408.

Pennycuick, C. J. 1969. The mechanics of bird migration. Ibis 111:525-556.

Pennycuick, C. J. 1975. Mechanics of flight. Pages 1-75 in Avian Biology, vol. 5 (D. S. Farner and J. R. King, Eds.). Academic Press, New York.

Pennycuick, C. J. 1985. Flight. Pages 218-223 in A Dictionary of Birds (B. Campbell and E. Lack, Eds.). T. and A. D. Poyser, Calton, United Kingdom.

Pennycuick, C. J. 1989. Bird Flight Performance. Oxford University Press, Oxford.

Peters, R. H. 1983. The Ecological Implications of Body Size. Cambridge University Press, United Kingdom.

RAYNER, J. M. V. 1988. Form and function in avian flight. Current Ornithology 5:1-66.

Remeš, V., AND T. E. MARTin. 2002. Environmental influences on the evolution of growth and developmental rates in Passerines. Evolution 56:2505-2518.

RickLeFs, R. E. 1976. Patterns of growth in birds. II. Growth rate and mode of development. Ibis 115:177-201.

RicKLEFS, R. E. 1983. Avian postnatal development. Pages 1-83 in Avian Biology, vol. 7 (D. S. Farner, J. R. King, and K. C. Parkes, Eds.). Academic Press, New York.

Roff, D. A. 1992. The Evolution of Life Histories: Theory and Analysis. Chapman and Hall Press, New York.

Savile, D. B. O. 1957. Adaptive evolution in the avian wing. Evolution 11:212-224.

Schmidt-Nielsen, K. 1984. Scaling: Why Is Animal Size So Important? Cambridge University Press, New York.

Schorger, A. W. 1966. The Wild Turkey: Its History and Domestication. University of Oklahoma Press, Norman.

Sibley, C. G., And J. E. Ahlquist. 1990. Phylogeny and Classification of Birds: A Study in Molecular Evolution. Yale University Press, New Haven, Connecticut.

Skutch, A. F. 1976. Parent Birds and Their Young. University of Texas Press, Austin.

Starck, J. M. 1993. Evolution of avian ontogenies. Current Ornithology 10:275-366.

Starck, J. M., AND R. E. Ricklefs, Eds. 1998. Avian Growth and Development: Evolution within the Altricial-Precocial Spectrum. Oxford University Press, New York.

Tobalske, B. W., and K. P. Dial. 2000. Effects of body size on take-off flight performance in the Phasianidae (Aves). Journal of Experimental Biology 203:3319-3332.

Tobalske, B. W., T. L. Hedrick, K. P. Dial, and A. A. Biewener. 2003. Comparative power curves in bird flight. Nature 421:363-366.

VARRICCHIO, D. J., F. JACKSON, J. J. BORKOWSKI, AND J. R. HORNER. 1997. Nest and egg clutches of the dinosaur Troodon formosus and the evolution of avian reproductive traits. Nature 385:247-250.

WARRICK, D. R. 1998. The turning- and linearmaneuvering performance of birds: The cost of efficiency for coursing insectivores. Canadian Journal of Zoology 76:1063-1079.

Warrick, D. R., and K. P. Dial. 1988. Kinematic, aerodynamic, and anatomical mechanisms in the slow, maneuvering flight of pigeons. Journal of Experimental Biology 201:655-672.

Warrick, D. R., K. P. Dial, AND A. A. Biewener. 1998. Asymmetrical force production in the slow maneuvering flight of birds. Auk 115:916-928. 